\title{
Cerebro-Umbilical Ratio at 30-34 weeks Gestation as A Prognostic Parameter for Pregnancy and Adverse Neonatal Outcomes
}

\author{
A.E.El Mashad , M.A.Mahmoud , A.S.Ebrahem and Y.A.Saeed \\ Obstetrics and Gynecology Dept., Faculty of Medicine, Benha Univ., Benha, Egypt \\ E-Mail:Yasoo.ahmed456@gmail.com
}

\begin{abstract}
Fetal cerebral dissemination normally shows a high opposition example of stream in the MCA. There is a typical physiologic reaction of MCA enlargement in babies presented to intense or ceaseless hypoxia, bringing about a reductionin fetal MCA PI. Umbilical corridor and center cerebral conduit Doppler ultrasound unmistakably delineate the data about placental obstruction and the adjustments in the fetal hemodynamics in light of it. The cerebral-umbilical proportion stays steady during the most recent 10 weeks of incubation and gives preferred symptomatic precision over either vessels pulsatility list (PI) alone. The current examination is forthcoming associate investigation which was completed at Benha University Hospital (outpatient center). The investigation included 90 pregnant ladies at 30-multi week incubation introduced for antenatal consideration and afterward followed till conveyance. There are factually huge relations between CUR level and gestational age, nearness of comorbid diabetes and hypertension, method of conveyance, APGAR score and NICU affirmation. There is measurably critical positive relationship among's CUR and both maternal age and APGAR score. The best cutoff of CUR in expectation of nonattendance of requirement for crisis $\mathrm{CS}$ is $\geq 0.94$. Additionally, the best cutoff of CUR in expectation of nonappearance of requirement for NICU affirmation is $\geq 1.0075$. Mutt is prescient of expanded obstetric intercession rates and unfriendly perinatal and neonatal results and might be helpful in certain high danger gatherings. Cerebro_umbilical proportion shows high exactness in contrast with center cerebral supply route and umbilical conduit Doppler alone so it is proficient test for anticipation and mediation.
\end{abstract}

Keywords: Doppler ultrasonography, Uteroplacental circulation (circle of Willis), Fetal hypoxemia, Cerebro-umbilical ratio.

\section{Introduction}

Doppler ultrasound is a valuable instrument for considering pathophysiological components that can influence the fetal hemodynamic status [1]. The assessment of intrauterine organs' perfusion and its connection with fetal status got conceivable, which is a useful strategy for fetal surveillance [2].

Doppler velocimetry is the best strategy for observation for fetal hypoxemia during pregnancy. Doppler ultrasound velocimetry of uteroplacental umbilical and fetal vessels has become a built up technique for antenatal observing, permitting the noninvasive appraisal of fetal flow. Its records give significant data on the hemodynamics of the vascularity of fetal vessels. Umbilical supply routes are the regular vessels evaluated, yet late investigations affirm the viability of center cerebral course (MCA) Doppler appraisal. MCA Doppler estimation is a notable methodology for distinguishing fetal compromise [3].

Doppler ultrasound velocimetry of uteroplacental umbilical and fetal vessels has become set up strategy for antenatal observing, permitting the non-intrusive evaluation of fetal circulation [4].

The upkeep of good utero-placental flow is important to proceed with an ordinary pregnancy. The movement of pregnancy is set apart by various changes and transformations in the maternal, placental and fetal vasculatures [5].

Helpless pregnancy result has indicated a solid connection with IUGR; the greater part the stillbirths are related with IUGR and about $10 \%$ of perinatal mortality is ensuing to undetected IUGR [6].

Umbilical conduit and center cerebral corridor doppler ultrasound plainly portray the data about placental opposition and the adjustments in the fetal hemodynamics because of it. Umbilical supply route Doppler mirrors the maldevelopment of the placental tertiary stem villi which expands the placental resistance [7].

MCA/UA proportion reflects not just the circulatory inadequacy of the umbilical velocimetry of the placenta showed by adjustments in the umbilical $\mathrm{S} / \mathrm{D}$ proportion yet in addition the versatile changes bringing about alterations of the center cerebral S/D ratio [8].

Doppler waveform variations from the norm have been accounted for to be the most exact indicator of poor neonatal outcome [9].

\section{Patients and methods}

This prospective, observational, clinical study was conducted in Department of Obstetrics \& Gynecology Benha University Hospital from February 2019 to March 2020. It comprised ninety singleton pregnant woman with gestational age between 30_34 weeks gestation from either the last normal menstrual period or by the earliest ultrasound examination or correlation with both.

Multiple pregnancies, known congenital malformations, non-cephalic presentation and premature rupture of membranes were excluded. 
All patients were subjected to complete history taking, general examination, abdominal examination and maternal weight and height.

Ultrasound was done by using LOGIQ p5 V machine with colour Doppler unit with transducer 3.5 $\mathrm{mHz}$ to assess gestational age determination, fetal presentation and placental site.

Doppler studies were done to assess umbilical artery Doppler indices [umbilical artery pulsatility index (UA-PI), umbilical artery resistance index (UA$\mathrm{RI}$ ), umbilical artery systolic/diastolic ratio (UA-S/D)], middle cerebral artery Doppler indices [middle cerebral artery pulsatility index (MCA-PI), middle cerebral artery resistance index (MCA-RI) , middle cerebral artery systolic/diastolic ratio (MCA - S/D)] and cerebro-umbilical ratio (ratio of MCA-PI / UA-PI).

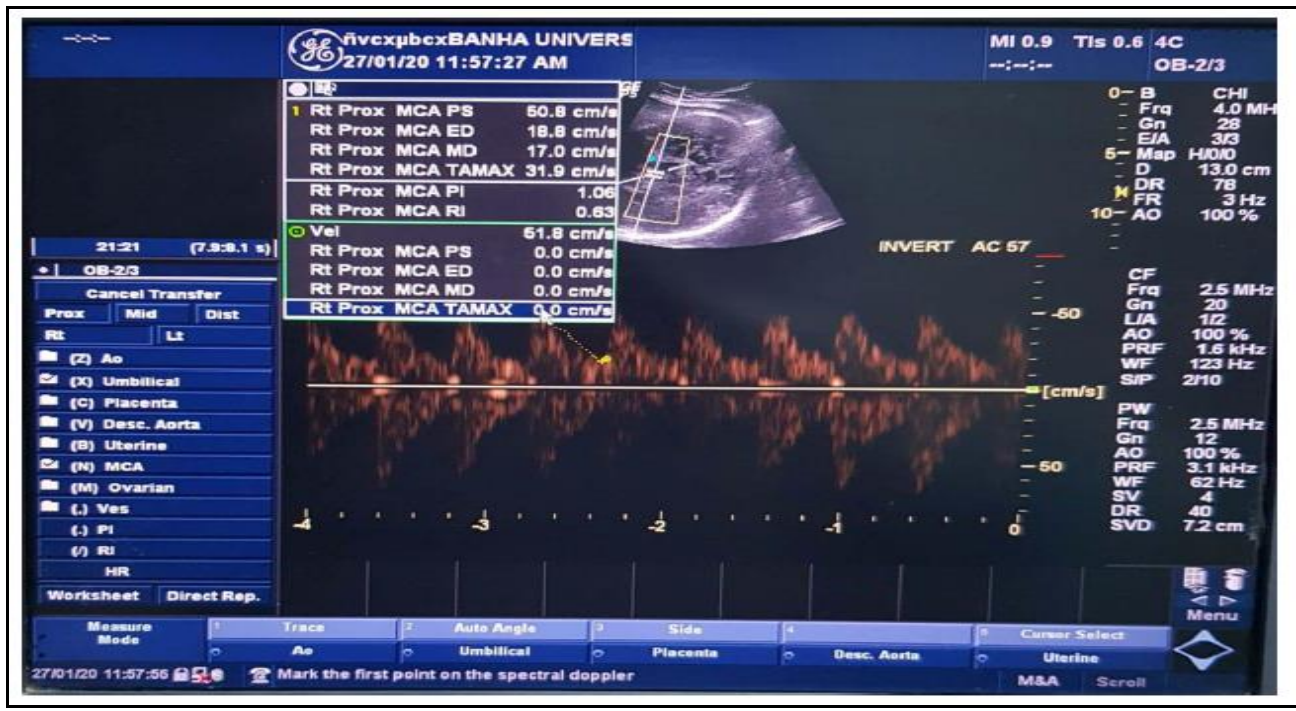

Fig (1) Color Doppler of the circle of Willis \& the middle cerebral artery.

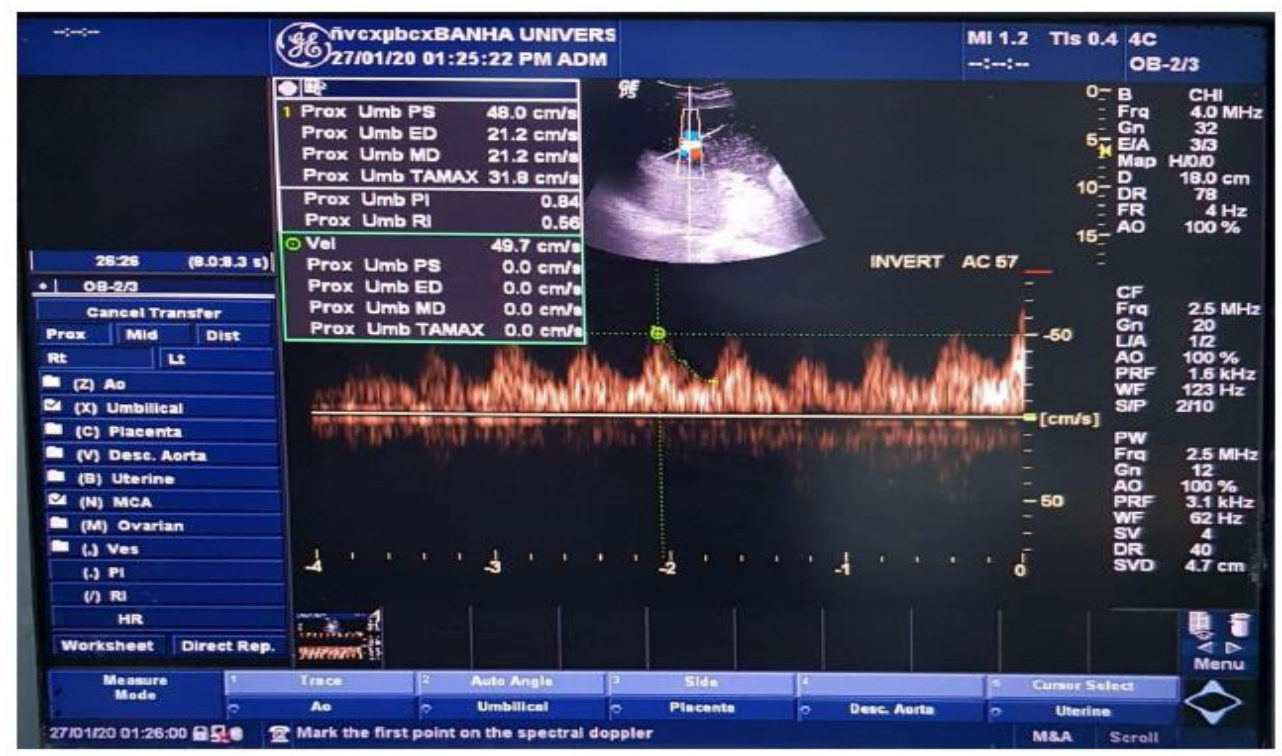

Fig (2) Doppler waveform of umbilical artery

Primary outcome was measurement of cerebroumbilical ratio. Secondary outcomes were pregnancy outcome (as regard mode of delivery) and neonatal outcomes (as regard APGAR score at 1 and 5 minutes, birth weight and admission to NICU ).

\section{Results}

Age of the studied participants ranged from 19 to 40 years with mean 26.6. gravidity, parity ranged from 1 to 6 and 0 to 3 with median 1 and 0 respectively. Ninety percent of patients were G1 and 2. About 52\% were primagravida and $77.8 \%$ had no previous history of abortion. Gestational age ranged from 30 o 34 weeks with mean 32.74 weeks. 
Table (1) Distribution of the studied patients according to baseline characteristics.

\begin{tabular}{|c|c|}
\hline & $\mathrm{N}=90$ \\
\hline Age & $26.6=6.72$ \\
\hline Mean $=$ SD & $19-40$ \\
\hline \multicolumn{2}{|l|}{ Range } \\
\hline Gravidity & $2.09=6.72$ \\
\hline Mean $=$ SD & 1 \\
\hline Median & $1 \_6$ \\
\hline Range & $63(70)$ \\
\hline$\leq 2$ & $27(30)$ \\
\hline \multicolumn{2}{|l|}{$<2$} \\
\hline Parity & $0.79=1.55$ \\
\hline Mean $=$ SD & 0 \\
\hline Median & $0 \_3$ \\
\hline Range & $47(\overline{5} 2.2)$ \\
\hline Primigravida & $23(47.8)$ \\
\hline \multicolumn{2}{|l|}{ From 1 to 3} \\
\hline Abortion & $0.3=0.61$ \\
\hline Mean = SD & 0 \\
\hline Median & $0 \_2$ \\
\hline Range & $70(77.8)$ \\
\hline No & $20(22.2)$ \\
\hline \multicolumn{2}{|l|}{$1-2$} \\
\hline Gestational age (weeks) & $32.74=1.32$ \\
\hline Mean = SD & $30 \_34$ \\
\hline Range & \\
\hline
\end{tabular}

CUR ranged from 0.8 t0 2.1 with mean 1.28 . about $68 \%$ had CUR more than 1 .

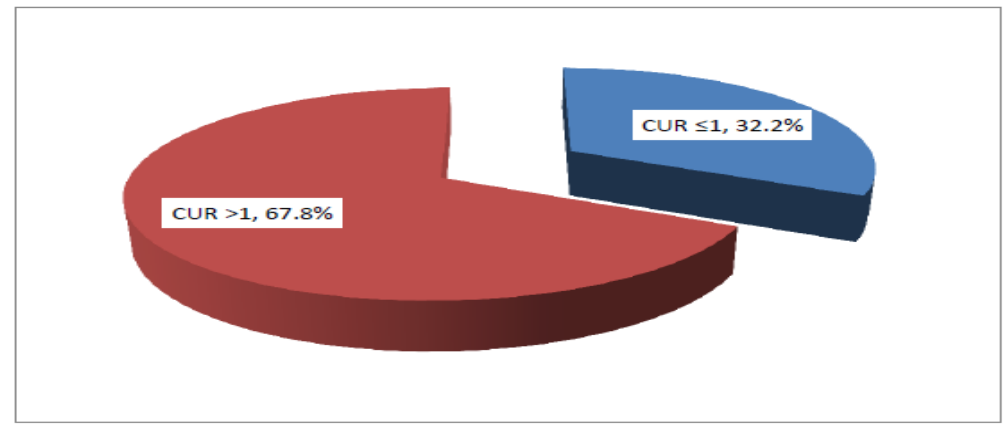

Fig (3) Pie chart showing distribution of the studied patients according to CUR level Regarding neonatal outcome, $35.6 \%$ of infants were admitted to NICU.

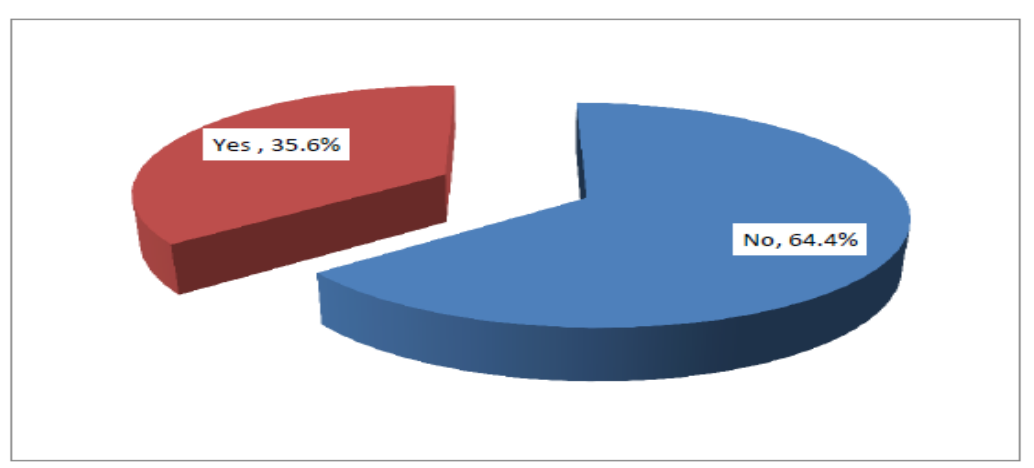

Fig (4) Pie chart showing distribution of the studied patients according to NICU admission 
There is statistically significant relation between need for NICU admission and presence of comorbid diabetes and hypertension. Thirty one percent of those admitted to NICU had maternal history of PIH $(\mathrm{p}<0.05)$. about $18 \%$ of those admitted to NICU had maternal history of diabetes.

Table (2) Relation between CUR and comorbidity of the studied patients.

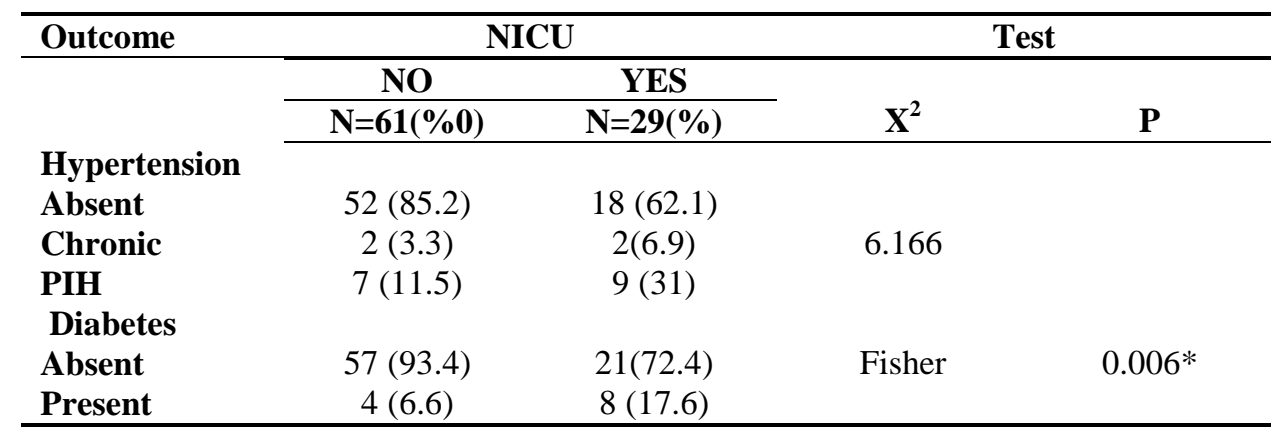

There is statistically significant relation between need for NICU admission and mode of delivery.

Table (3) Relation between NICU admission and mode of delivery of the studied patients.

\begin{tabular}{lcccc}
\hline Mode of delivery & \multicolumn{2}{c}{ NICU } & \multicolumn{2}{c}{ Test } \\
\cline { 2 - 3 } & $\mathbf{N o}$ & Yes & $\mathbf{X}^{\mathbf{2}}$ & P \\
\cline { 2 - 3 } & $\mathbf{N}=\mathbf{6 1}(\%)$ & $\mathbf{N = 2 9 ( \% )}$ & & \\
\hline Vaginal delivery & $33(54.1)$ & $0(0)$ & 49.389 & $<0.001^{* *}$ \\
Elective CS & $0(0)$ & $13(44.8)$ & & \\
Emergency CS & $28(45.9)$ & $16(55.2)$ & & \\
\hline
\end{tabular}

There is statistically significant relation between CUR level and presence of comorbid diabetes and hypertension. Larger percentage of participants with comorbid hypertension and diabetes had CUR $\leq 1$.

Table (4) Relation between CUR and comorbidity of the studied patients.

\begin{tabular}{lccccc}
\hline Outcome & \multicolumn{3}{c}{ Cerebro-umbilical ratio } & Test & \\
\cline { 2 - 5 } & Total & $\begin{array}{c}\text { CUR } \leq \mathbf{1} \\
\mathbf{N = 2 9}(\%)\end{array}$ & $\begin{array}{c}\text { CUR>1 } \\
\mathbf{N = 6 1}(\%)\end{array}$ & $\mathbf{X}^{\mathbf{2}}$ & P \\
\hline Hypertension & & & & & \\
Absent & 70 & $18(25.7)$ & $52(74.3)$ & 6.166 & $0.046^{*}$ \\
Chronic & 4 & $2(50)$ & $2(50)$ & & \\
PIH & 16 & $9(56.3)$ & $7(43.7)$ & & $0.001^{*}$ \\
Diabetes & & & & Fisher & \\
Absent & 78 & $20(25.6)$ & $58(74.4)$ & & \\
Present & 12 & $9(75)$ & $3(25)$ & & \\
\hline
\end{tabular}

There is statistically significant relation between CUR level and mode of delivery. All participants delivered by emergency CS had CUR $\leq 1$.

Table (5) Relation between CUR and mode of delivery of the studied patients.

\begin{tabular}{lccccc}
\hline Mode of delivery & \multicolumn{3}{c}{ Cerebro-umbilical ratio } & \multicolumn{2}{c}{ Test } \\
\cline { 2 - 5 } & Total & $\begin{array}{c}\text { CUR } \leq \mathbf{1} \\
\mathbf{N}=\mathbf{2 9}(\boldsymbol{\%})\end{array}$ & $\begin{array}{c}\text { CUR>1 } \\
\mathbf{N}=\mathbf{6 1}(\boldsymbol{\%})\end{array}$ & $\mathbf{X}^{\mathbf{2}}$ & $\mathbf{P}$ \\
\hline Vaginal delivery & 33 & $3(9.1)$ & $30(90.9)$ & 42.892 & $<0.001^{* *}$ \\
Elective CS & 41 & $10(24.4)$ & $31(75.6)$ & & \\
Emergency & 16 & $16(100)$ & $0(0)$ & & \\
\hline
\end{tabular}

There is statistically significant relation between CUR level and APGAR score (higher in participants with CUR>1). Larger percentage of participants admitted to NICU $(89.7 \%)$ had CUR $\leq 1$. 
Table (6) Relation between CUR and baseline characteristics of the studied patients.

\begin{tabular}{|c|c|c|c|c|c|}
\hline \multirow[t]{2}{*}{ Outcome } & \multicolumn{3}{|c|}{ Cerebro-umbilical ratio } & \multicolumn{2}{|c|}{ Test } \\
\hline & Total & $\mathrm{CUR} \leq 1$ & CUR>1 & $\mathrm{T} / \%^{2}$ & $\mathbf{P}$ \\
\hline APGAR & & $\mathrm{N}=29(\%)$ & $\mathrm{N}=61(\%)$ & & \\
\hline Mean=SD & & $6.41=0.95$ & $8.57=1.07$ & -9.693 & $<0.001 * *$ \\
\hline Range & & $6-9$ & $6-10$ & & \\
\hline NICU adm & & & & & \\
\hline Yes & 29 & $26(89.7)$ & $3(10.3)$ & Fisher & $<0.001 * *$ \\
\hline NO & 61 & $3(4.9)$ & $58(95.1)$ & & \\
\hline
\end{tabular}

There is statistically significant positive correlation between CUR and maternal age and
APGAR score (higher in participants with CUR>1). There is statistically non-significant correlation between CU R and gestational age.

Table (7) Correlation between CUR and maternal age, gestational age and APGAR score.

\begin{tabular}{lcc}
\hline Factors & \multicolumn{2}{c}{ CUR } \\
\cline { 2 - 3 } & $\mathbf{R}$ & $\mathbf{P}$ \\
\hline Maternal age (years) & 0.314 & $0.004^{*}$ \\
Gwstational age ( weeks) & 0.018 & 0.866 \\
Apgar Score & 0.373 & $<0.001^{* *}$ \\
\hline
\end{tabular}

The best cutoff of CUR in prediction of need for emergency CS is $\geq 0.94$ with area under curve 0.892 , sensitivity $89.2 \%$, specificity $50 \%$, positive predictive value (PPV) $89.2 \%$, negative predictive value (NPV) $50 \%$ and accuracy $79.3 \%(\mathrm{p}<0.05)$.

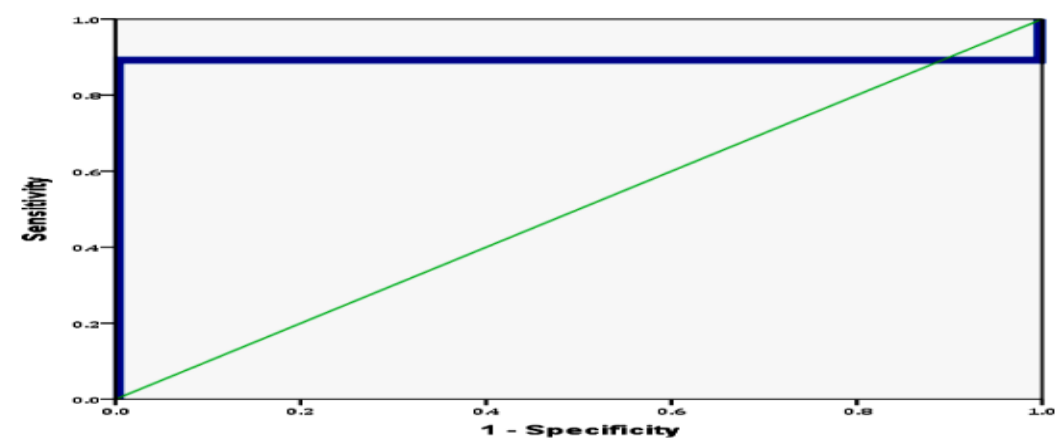

Fig (5) ROC curve showing performance of CUR in prediction of need for emergency CS among the studied patients.

The best cutoff of CUR in prediction of need for emergency CS is $\geq 1.0075$ with area under curve 0.985 , sensitivity $95.1 \%$, specificity $89.7 \%$, positive predictive value (PPV) 95.1\%, negative predictive value (NPV) $89.7 \%$ and accuracy $93.3 \%(\mathrm{p}<0.05)$.

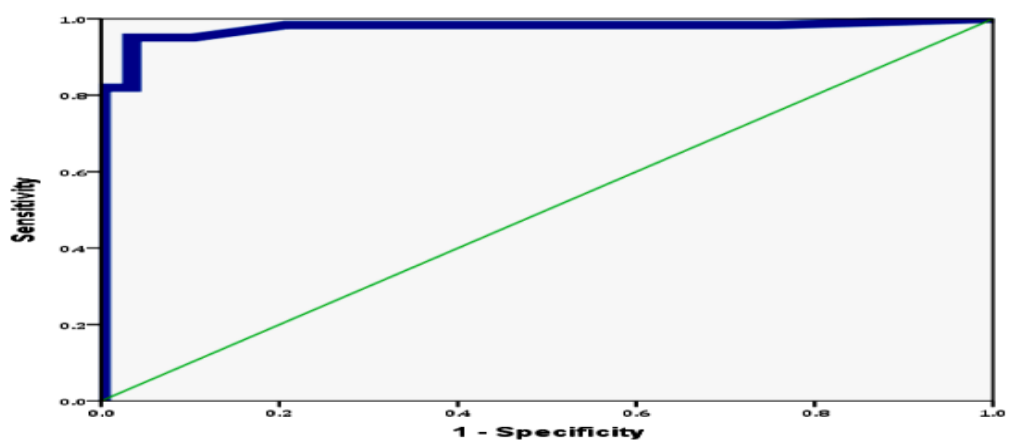

Fig (6) ROC curve showing performance of CUR in prediction of poor neonatal outcome (NICU admission) among the studied patients. 


\section{Discussion}

A low CUR estimated before in pregnancy is related with expanded paces of antagonistic pregnancy results including expanded intrapartum intercession rates [10].

Albeit a few instances of intra-partum fetal hypoxia-ischemia might be hastened by intense disastrous occasions, for example, rope prolapse or uterine crack, by and large hypoxemia grows progressively. Embryos with diminished feto-placental stores before work have a diminished capacity to endure the ordinary yet dynamic hypoxic stress brought about by intrapartum uterine withdrawals which lessen uterine blood stream by as much as $60 \%$.

The CUR reflects problematic placental capacity and related fetal cardiovascular pay and seems, by all accounts, to be a superior indicator of result than either the UA PI or MCA PI individually [11].

The point of the investigation was to assess the connection between cerbro-umbilical proportion at 3034 weeks development with pregnancy and unfavorable neonatal results.

The current examination is imminent , observational, clinical investigation which was done at Benha University Hospital. The examination included 90 pregnant ladies between 30-multi week growth introduced for antenatal consideration and afterward followed till conveyance.

Age of the examined members extended from 19 to 40 years with mean 26.6. Gravidity, equality went from 1 to 6 and 0 to 3 with middle 1 and 0 separately. A lot of patients were G1 and 2. About 52\% were primagravida and $77.8 \%$ had no past history of fetus removal. Gestational age ran from 30 to 34 weeks with mean 32.74 weeks. About $22 \%$ and $13 \%$ of the considered members had comorbid hypertension and diabetes separately.

As per Doppler estimation, MCA PI ran from 0.75 to 1.45 with mean 1.07 and UA PI ran from 0.56 to 1.33 with mean 0.87 [12]. found that babies with unfavorable result had a lower center cerebral corridor PI record.

In our investigation, CUR ran from 0.8 to 2.1 with mean 1.28. about 68\% had CUR mutiple [13] explored the prescient estimation of the CUR estimated at 30-34 weeks for unfavorable pregnancy results. The CUR seemed, by all accounts, to be better than the MCA PI or UA PI alone for the forecast of crisis cesarean segment for fetal trade off. Newborn children with a CUR $<1$ were right around multiple times bound to be conveyed by crisis cesarean area for fetal trade off than conveyed vaginally. In any case, in spite of the unmistakable affiliation and the high certain prescient worth, the general discovery rates were low.

M.Ropacka-Lesiak et al.[14] evaluated the value of $\mathrm{C} / \mathrm{U}$ in expectation of the intrapartum FHR anomalies and the antagonistic perinatal result in simple pregnancies at 40 weeks and past. They have indicated that a low CUR is intelligent of problematic placental capacity and going with fetal cardiovascular remuneration and that it is a superior indicator than the MCA PI or UA PI alone for a scope of obstetric and neonatal results. These antagonistic results incorporate fetal development limitation, expanded intrapartum usable rates, expanded neonatal unit affirmation and helpless corrosive base status during childbirth. They presumed that the $\mathrm{C} / \mathrm{U}$ record shows the most elevated affectability in forecast of FHR anomalies and unfriendly neonatal result in straightforward pregnancies at 40 weeks and past. The $\mathrm{C} / \mathrm{U}$ list is valuable in clinical practice in antenatal observing of these ladies so as to choose those at high danger of intra-and baby blues confusions.

Likewise, [15] indicated that babies showing diminished development speed over the third trimester yet in this way brought into the world with birth loads inside the typical range, had low CURs and clinical highlights of placental inadequacy and were at expanded danger of stillbirth. In a bigger report, 16) found that a low CUR was additionally an autonomous danger factor for stillbirth and perinatal.

S.Twomey, [17] explored the connection between the cerebro-umbilical proportion (CUR), estimated at 30-34 weeks, and antagonistic intrapartum and perinatal results. A sum of 1224 ladies were remembered for the investigation. Generally speaking, $6.1 \%(75 / 1224)$ of the investigation accomplice had a CUR $<1$.

Deora and Gara [12] assessed the anticipating estimation of the MCA/UA proportion, for fetal guess in ladies with gestational hypertension and toxemia, twin pregnancy, post-dated pregnancy. They proposed that the MCA/UA PI Doppler proportion of under 1 was a decent prescient device for neonatal result in preeclamptic and hypertensive pregnant ladies and could be utilized to distinguish hatchlings in danger of dismalness and mortality. The utilization of Doppler ultrasound in high-hazard pregnancies seems to improve various obstetric consideration results and promising in diminishing perinatal passings.

In our investigation, there is measurably critical connection between CUR level and gestational age. [12] found that babies with strange Doppler MCA/UA PI proportion had a fundamentally lower gestational age at conveyance.

In our examination, there is factually critical connection between CUR level and nearness of comorbid diabetes and hypertension (bigger level of members with comorbid hypertension and diabetes had CUR $\leq 1$ ) [17] found that ladies in the CUR $<1$ had essentially more regrettable perinatal results including higher paces of preterm birth, low birth weight, higher paces of respiratory misery and hypoglycemia.

In our examination, there is measurably noteworthy connection between CUR level and method of conveyance (all members conveyed by crisis CS had CUR $\leq 1$ [17] found that the CUR was fundamentally lower in the partner of ladies who required a crisis cesarean segment for fetal trade off contrasted with every single other gathering. 
Dog < 1, estimated at 30-34 weeks, is related with a more serious danger of crisis cesarean conveyance for fetal trade off and various other antagonistic perinatal results. The affiliation was most grounded in low birth weight children.

In our examination, there is measurably critical connection between CUR level and APGAR score (higher in members with CUR>1) [17]. found that ladies in the CUR $<1$ accomplice had fundamentally more awful perinatal result including discouraged 5 min Apgar scores during childbirth. (12) found that embryos with irregular Doppler MCA/UA PI proportion had an altogether lower Apgar score at 5minute.

In our examination, there is authentically gigantic association between CUR level and NICU certification (greater degree of individuals admitted to NICU had CUR $\leq 1)$. (12) found that hatchlings with weird Doppler MCA/UA PI extent had a basically higher event of admission to NICU.

In our investigation, there are measurably noncritical relations between CUR level and maternal age, gravidity, equality or fetus removal. 17) found a higher extent of 129 nulliparous $(\mathrm{p}=0.011$ ) ladies and ladies with hypertension in the CUR $<1$ companion contrasted with the CUR $>1$ gathering.

In our investigation, there is measurably huge positive relationship among's CUR and both maternal age and APGAR score. There is factually nonnoteworthy connection among's CUR and gestational age.

Doppler information consolidating both umbilical and cerebral velocimetry give extra data on fetal outcomes of the placental anomaly. Unusual MCA/UA PI Doppler proportion is emphatically associated with more regrettable fetal anticipation. In typical pregnancies, the diastolic segment in the cerebral conduits is lower than in the umbilical courses at any gestational age. In this way, the cerebro-vascular obstruction stays higher than the placental opposition and the MCA/UA proportion is more noteworthy than 1. The file turns out to be under 1 if the stream circulation is supportive of the mind in obsessive pregnancies [18].

In our investigation, the best cutoff of CUR in forecast of nonappearance of requirement for crisis CS is $\geq 0.94$ with zone under bend 0.892 , affectability $89.2 \%$, particularity half, positive prescient worth (PPV) 89.2\%, negative prescient worth (NPV) half and precision $79.3 \%(\mathrm{p}<0.05)$.

Additionally, the best cutoff of CUR in forecast of nonappearance of requirement for NICU confirmation is $\geq 1.0075$ with territory under bend 0.985 , affectability $95.1 \%$, particularity $89.7 \%$, positive prescient worth (PPV) 95.1\% , negative prescient worth (NPV) $89.7 \%$ and precision $93.3 \%(\mathrm{p}<0.05)$. D.Arduini, [19] found that UA/MCA PI list proportion was the best test when contrasted and MCA, UA, and RA PI records (affectability 89\%, explicitness 94\%).
As to administrator bend (ROC) investigation utilizing the MCA PI or UA PI for the forecast of any crisis cesarean conveyance, 17) exhibited a region under the bend (AUC) of 0.62 and 0.60 individually. The AUC for the CUR was somewhat better at 0.63. The unadjusted OR for the danger of crisis cesarean conveyance for a CUR $<1$ was 18.1 . The recognition rate (DR) for CUR $<1$ as an indicator for any crisis cesarean conveyance was $13.4 \%$ with a bogus positive rate (FPR) of $0.85 \%$, a positive prescient worth (PPV) of $92.0 \%$ and a negative prescient worth (NPV) of $61.2 \%$. The DRs expanded essentially to $47.4 \%$ and $59.0 \%$ for children with birth loads < 10 th centile and $<5$ th centile individually. Additionally, the DR expanded to $23.8 \%$ for children conceived inside about fourteen days of the output date.

Be that as it may, with respect to ROC examination utilizing the MCA PI or UA PI for the forecast of crisis cesarean conveyance for fetal trade off, 17) exhibited an AUC of 0.64 and 0.67 separately. The AUC for the CUR was 0.69. The unadjusted OR for the danger of crisis cesarean conveyance for fetal trade off for a CUR $<1$ was 27.4. The DR for CUR $<1$ as an indicator for crisis cesarean conveyance for fetal trade off was $18.9 \%$ with a FPR of $0.85 \%$, a PPV of $80.6 \%$ and a NPV of $86.8 \%$. The DRs expanded to $45.5 \%$ and $61.1 \%$ for children with birth loads $<10$ th centile and $<5$ th centile separately. For children conceived inside about fourteen days of the output date, the DR expanded to $41.7 \%$.

R.Deora, [12] discovered high affectability of the MCA/UA PI proportion in foreseeing stillbirth (100\%). Likewise, they found a high explicitness and positive anticipating an incentive deprived for therapy in neonatal emergency unit.

Despite the fact that there is a reasonable relationship between a low CUR and unfriendly results, the identification rates and bogus positive rates are presently imperfect. Mutt might be more helpful for the evaluation of hatchlings recognized to be little for gestational age who are known to be at expanded danger for an assortment of unfriendly outcomes(20).

G.R.DeVore, [11] has utilized differing shorts including centiles $(<2.5$ th, $<5$ th 230 or $<10$ th centiles $)$, total qualities $(<1$ or $<1.08)$ or products of 231 middle (MoM) $(<0.05$ or $<0.6765 \mathrm{MoM})$.

Thusly, CUR is prescient of expanded obstetric intercession rates and unfavorable perinatal and neonatal results and might be helpful in certain high danger gatherings.

Cerebro-umbilical proportion shows high exactness in contrast with center cerebral vein and umbilical corridor doppler alone so it is productive test for anticipation and intercession.

\section{References}

[1] D. Mihu, D.Diculescu, N.Costin Applications of Doppler ultrasound during labor. Medical Ultrasonography, Vol.13(2), PP.141,2011. 
[2] K.Konje, R.Abrams , D.J.Taylor. Normative values of Doppler velocimetry of five major fetal arteries as determined by color power angiography. Acta Obstetricia et Gynecologica Scandinavica, Vol.84(3),pp.230-237,2005.

[3] R.Najam, G.Sarika , H.Shalini. Predictive Value of Cerebroplacental Ratio in Detection of Perinatal Outcome in High-Risk Pregnancies. The J.,Obstetrics and Gynecology of India, Vol.66(4),pp.244-247,2016.

[4] M.Dubiel, G.H.Breborowicz, K.Marsal. Fetal adrenal and middle cerebral artery in high risk pregnancy. Ultrasound Obstet Gynecol,Vol.16, PP.414-8,2000.

[5] A.J.du Plessis Cerebral blood flow and metabolism in the developing fetus. Clin Perinatol,Vol.36,pp.531-548,2009.

[6] J.F.Froen, J.O.Gardosi, A.Thurmann. Restricted fetal growth insudden intrauterine unexplained death. Acta Obstet Gynecol Scand,Vol.83,pp.801$807,2004$.

[7] S.Mohan, P.Natarajan, S.Madineni. Study of Triple Vessel Wave Pattern by Doppler Studies in Low Risk and High Risk Pregnancies and Perinatal Outcome. IOSR J.,Dental and Medical Sciences,Vol.16, PP.14-23,2017.

[8] G.Sterne, L.E.Shields, T.J.Dubinsky. Abnormal fetal cerebral and umbilical Doppler measurements in fetuses with intrauterine growth restriction predicts the severity of perinatal morbidity. J Clin Ultrasound,Vol.29,PP.146-51,2001.

[9] L.M.McCowan. A pilot randomized controlled trial of two regimens of fetal surveillance for small for gestational age fetuses with normal results of umbilical artery doppler velocimetry. Am J Obstet Gynecol,Vol.182(1 Pt 1), PP.81-6. ,2000.

[10]R. Akolekar, A. Syngelaki, D.M.Gallo. Umbilical and fetal middle cerebral 296 artery Doppler at 35-37 weeks' gestation in the prediction of adverse perinatal 297 outcome. Ultrasound Obstet Gynecol,Vol.46(1),PP.82-92,2015.

[11] G.R.DeVore. The importance of the cerebroplacental ratio in the evaluation of 305 fetal well-being in SGA and AGA fetuses. Am J Obstet Gynecol,Vol.213(1),PP.306 5-15,2015.

[12] R.Deora, N.Gara. The value of the middle cerebral to umbilical artery doppler ratio in the prediction of neonatal outcome in patients with high risk pregnancy. Int $\mathrm{J}$ Reprod Contracept Obstet Gynecol,Vol.6, PP.3965-8,2017.

[13] S.Bakalis, R.Akolekar, D.M.Gallo. Umbilical and fetal middle cerebral artery Doppler at 30-34 weeks' gestation in the prediction of adverse perinatal outcome. Ultrasound Obstet Gynecol,Vol.45, PP.409-20,2015.

[14] M.Ropacka-Lesiak, T.Korbelak, J. S'widerMusielak. Cerebro-placental ratio in prediction of adverse perinatal outcome and fetal heart rate 318 disturbances in uncomplicated pregnancy at 40 weeks and beyond. Arch Med Sci,Vol.11(1), PP.142-8,2015.

[15]N. Bardien, C.L.Whitehead, S.Tong. Placental insufficiency in fetuses that slow in growth but are born appropriate for gestational age: a prospective longitudinal study. PLoS One, Vol.11, PP.2016.

[16] A, Khalil, J.Morales-Rosello, R.Townsend. Value of third-trimester cerebroplacental ratio and uterine artery Doppler indices as predictors of stillbirth and perinatal loss. Ultrasound Obstet Gynecol,Vol.47, PP.74-80,2016.

[17] S.Twomey, C.Flatley , S.Kumar. The association between a low cerebro-umbilical ratio at 30-34 weeks gestation, increased intrapartum operative intervention and adverse perinatal outcomes. European J.,Obstetrics and Gynecology and Reproductive Biology, Vol.50, PP.314-320,2016.

[18]R.O.Bahado-Singh, E.Kovanci, A.Jeffres. The Doppler cerebroplacental ratio and perinatal outcome in intrauterine growth restriction. Am J Obstet Gynecol,Vol.180, PP.750-6,1999.

[19]D.Arduini, G.Rizzo, C.Romanini. Changes of pulsatility index from fetal vessels preceding the onset of late decelerations in growth retarded fetuses. Obstet Gynecol,Vol.79, PP.605-10.1992.

[20] M.Damodaram, L.Story, E. Kulinskaya. Early adverse perinatal complications in preterm growth-restrcited fetuses. Aust NZJ Obstet Gynaecol,Vol.51,PP.204-209,2011.

[21]E.M.Ashraf. El.Mashad, A.Mahmoud Mahmoud, Ahma S.Ebrahem, A.S Yasmeen. Taha. CerebroUmbilical Ratio at 30-34 weeks Gestation as A Prognostic Parameter for Pregnancy and Adverse Neonatal Outcomes. BMFJ, Vol.25(5), PP.513525, 2020. 